\title{
ANFÍPODE SUBTERRÂNEO DO GÊNERO Spelaeogammarus COMO UM INDICADOR DE CONECTIVIDADE EM UM AQUÍFERO CÁRSTICO DA BACIA DO RIO SALITRE, CENTRO NORTE DO ESTADO DA BAHIA.
}

André Vieira de Araújo ${ }^{1}$; Luiz Rogério Bastos Leal \& Doriedson Ferreira Gomes

Resumo - A distribuição da espécie Spelaeogammarus trajanoae, Crustáceo aquático da Ordem Amphipoda foi determinada em sete cavernas distribuídas no aquífero cárstico da Bacia do rio Salitre. Os crustáceos encontrados habitam exclusivamente água subterrânea. Essa distribuição localizada pode sugerir uma dispersão na direção do fluxo dentro do aquífero. Essa fauna que é restrita as águas subterrâneas podem ser ferramentas uteis para traçar caminhos do fluxo sem a necessidade do uso de corantes.

\begin{abstract}
The distribution of the species Spelaeogammarus trajanoae, water Crustacean of the Amphipoda Order was determined in seven caves distributed in the karst aquifer of the river Salitre Basin. The crustaceans found exclusively inhabit groundwater. This localized distribution may suggest a dispersion in the flow direction within the aquifer. This fauna that is restricted groundwater can be useful tools to trace the flow paths without the use of dyes.
\end{abstract}

Palavras-chave: Fluxo de água subterrânea; Carste; Crustáceo.

\section{INTRODUÇÃO}

Carste é um tipo de paisagem em que a troca entre as águas subterrâneas e superficiais resultam em um complexo e imprevisível aquífero (Bonacci et al., 2009). Os aquíferos cársticos são caracterizados do ponto de vista hidrogeológico por uma complexa circulação de água, com anomalias nas direções de fluxo de água com relação ao gradiente potenciométrico regional e muita variação dos valores de coeficiente de armazenamento e de transmissividade dentro do mesmo aquífero (Silva, 2002). A caracterização hidrogeológica do aquífero implica na compreensão da distribuição espacial do fluxo de água e de armazenamento e como estes variam ao longo do tempo.

\footnotetext{
${ }^{1}$ NEHMA /UFBA - Instituto de Geociências - Universidade Federal da Bahia Campus Ondina - Salvador (BA) - CEP 40210-340 Tel: (71) 32838534 - Fax: (71) 3283 8501, e-mail: anddrevieira@gmail.com
} 
Tradicionalmente testes com traçadores químicos são utilizados para desenvolver modelos conceituais de como as massas de água subterrânea estão ligadas entre si (Maurice et al., 2011). Testes com traçadores fornecem informações sobre partes inacessíveis e inexploradas dentro do sistema cárstico (Goldscheider et al., 2008).

Eles são considerados como o meio mais confiável e eficiente para recolher informações hidráulicas da subsuperficie cárstica (Campo, 1999). Smart e Worthington (2004) classificam os traçadores em físicos, químicos, isotópicos e biológicos.

Pipan e Culver (2007) usaram crustáceos Copepoda como traçadores biológicos no epicarste (a parte mais superficial do carste). A vantagem de usar os organismos como traçadores é que são naturais e já presentes no sistema, sem a necessidade para introdução de traçadores (corantes e ou sais) possivelmente prejudiciais que perubam o meio ambiente. Além das medidas quantitativas, os traçadores biológicos tambem fornecem informações qualitativas sobre o tipo de conexão entre as águas e a implicação para a dispersão de organismos. (Dole-olivier e Marmonier, 1992; Hahn, 2006; Arietti e Edwards, 2006).

Spelaeogammarus é um gênero pertencente à família Artesiidae e ocorre exclusivamente nas águas subterrâneas do Estado da Bahia. Atualmente são conhecidas seis espécies desse gênero, todas foram encontradas em cavernas, entre estas está $S$. trajanoae que é endêmica das cavernas calcárias na bacia do rio Salitre; (Bastos-Pereira e Ferreira 2015; Senna et al., 2014; Koenemann \& Holsinger 2000). Por serem espécies endêmicas a distribuição dos anfípodes do gênero Spelaeogammarus provavelmente é o resultado de uma colonização por uma população ancestral em uma única localidade com subsequente dispersão seguindo a direção do fluxo da água subterrânea no aquífero. Este trabalho explora a possibilidade de usar anfípodes subterrâneos como um traçador natural da conectividade entre as águas subterrâneas nas cavernas do sistema cárstico da bacia do rio Salitre.

\section{2. ÁREA DE ESTUDO}

A ênfase desse trabalho é a região cárstica da bacia do rio Salitre. Localizada no centro-norte do estado da Bahia, a bacia hidrográfica do rio Salitre é uma sub-bacia do Rio São Francisco. (Figuras 1 e 2). 


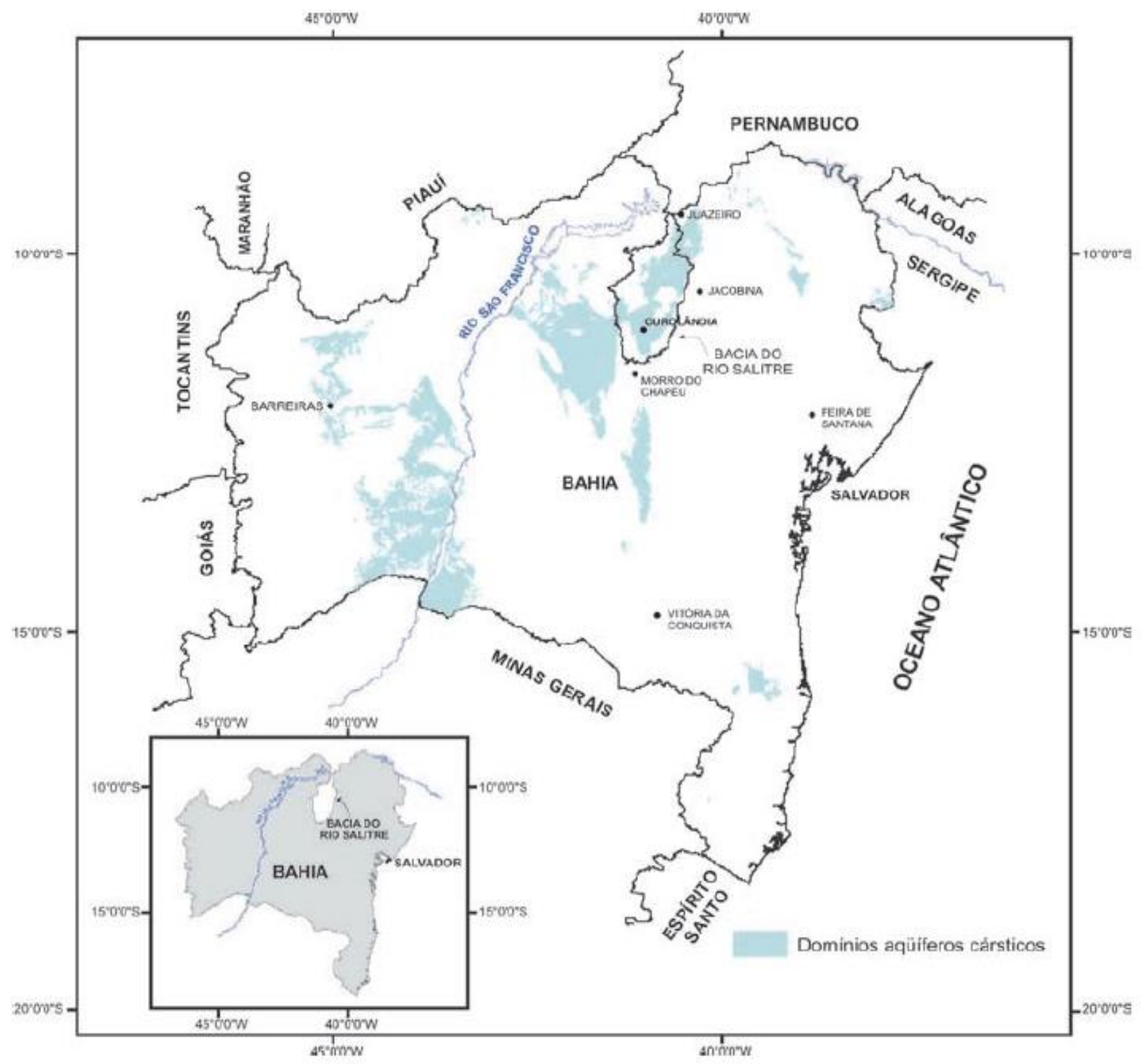

Figura 1. Localização da bacia hidrográfica do rio Salitre e dos domínios aquíferos cársticos do Estado da Bahia. 


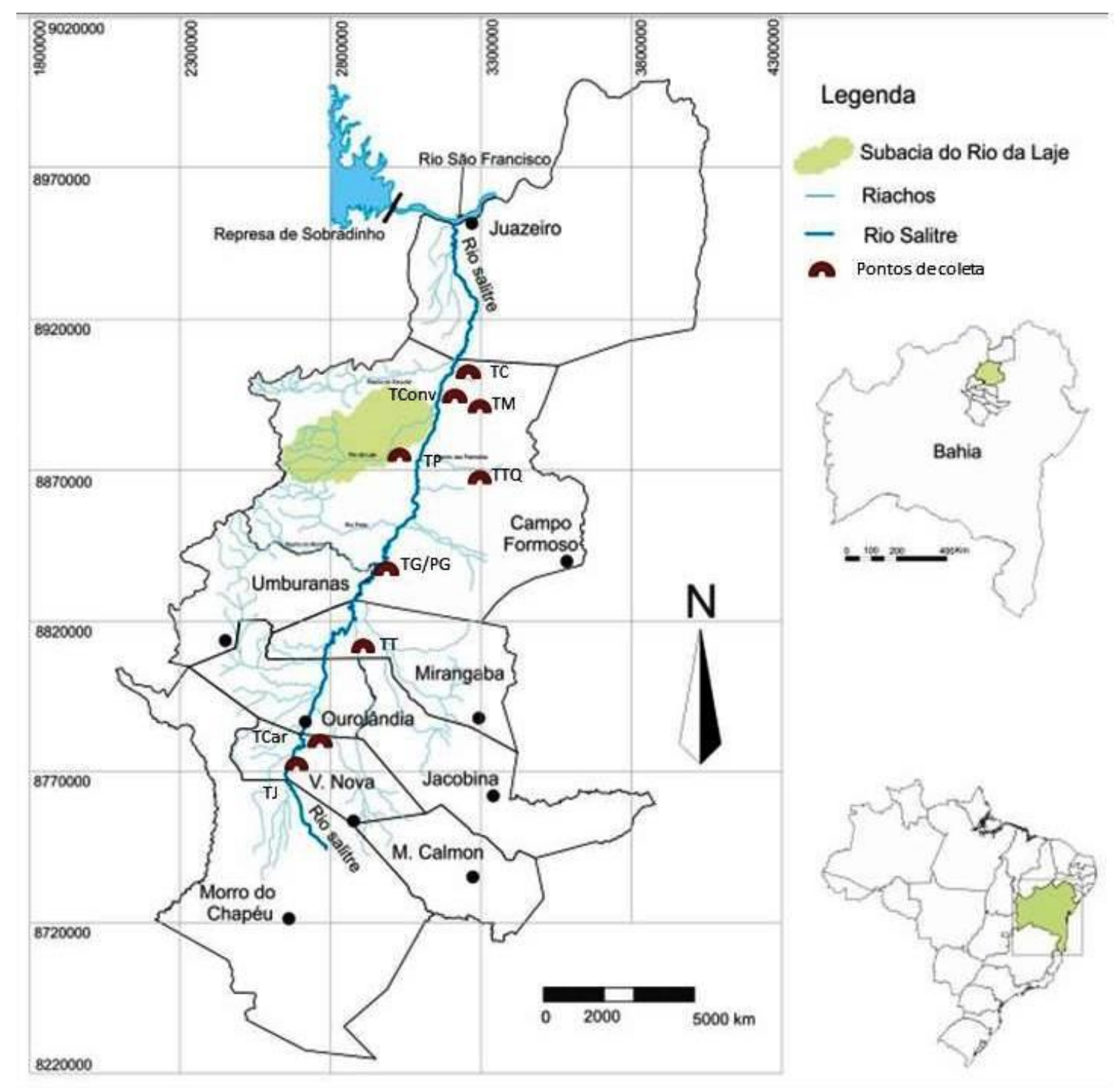

Figura 2. Bacia do rio Salitre, limites dos municípios e pontos de coleta (Adaptado de Silva 2009).

A bacia hidrográfica do rio Salitre, situada no centro - norte do Estado da Bahia possui uma área de $13.467,93 \mathrm{~km}^{2}$. A Bacia é dividida em três sub- regiões do sul para o norte: Alto, Médio e Baixo Salitre.

A bacia apresenta forma estreita e alongada e a rede de drenagem é formada por rios intermitentes como consequência da irregularidade das chuvas. Seu curso principal, o rio Salitre nasce na boca da madeira no município de morro do chapéu e escoa na direção norte - sul, indo desaguar no rio São Francisco, no município de Juazeiro percorrendo um total de 333, 24 km. 
$\mathrm{Na}$ bacia, as rochas do Proterozóico superior (Grupo Una: formação bebedouro e formação salitre) e coberturas calcárias cenozoicas (Formação Caatinga) são denominados de domínio de aquífero cárstico (Ribeiro, 2015).

A coleta de água subterrânea para os testes hidroquímicos foram feitos em 16 pontos sendo 8 em cavernas , 4 em poços tubulares e 4 em águas superficiais; Para os dados biológicos foram amostrados as águas superficiais e das cavernas distribuídas na região do alto, médio e baixo rio Salitre nos meses de outubro de 2015 e Abril de 2016. No alto Salitre foram feitas amostras nas cavernas: Toca do Carlito (Tcar), Toca da Jurema (TJ) e Toca da Trincheira (TT); No médio Salitre foram feitas amostras na Toca do Pitu (TP) e Toca da Tiquara (TTQ); No baixo Salitre as amostras foram amostradas água na, Toca do Martiliano (TM), Toca do Convento (TConv) e Toca do Cesário (TC).

\section{MATERIAIS E MÈTODOS}

Foram analisados in situ os Parâmetros físico-químicos: Temperatura, Salinidade, Condutividade, Ph, Oxigênio dissolvido. Com auxilio de sonda multiparâmetros da marca Horiba;

Nutrientes inorgânicos e orgânicos dissolvidos: Amônio, Nitrito, Nitrato, fosfato, sulfatos, silicatos, carbonatos, bicarbonatos, sódio, cloro, magnésio e cálcio. Analisados através da técnica de Espectrometria de massas com plasma indutivamente acoplado (ICP -MS).

As coletas biológicas realizadas através de métodos de amostragens visuais com redes de arrasto e de coleta de plâncton com malhas de $60 \mu$, acondicionados em potes plásticos com solução Transeau (6 partes de água destilada: 3 de álcool etílico 92º 1 de Formol).

Os diagramas hidroquímicos foram feitos utilizando o Software Qualigraf (FUCEME) e os mapas de fluxo realizados com o software Surfer versão 9.

\section{DADOS HIDROQUÍMICOS}

O tratamento hidroquímico dos dados disponíveis revelou através da distribuição dos cátions e ânions maiores, utilizando-se os diagramas de classificação de Piper e Stiff que as águas subterrâneas dos nove pontos amostradas no domínio aquífero cárstico da bacia são 
classificadas predominantemente como cloretadas cálcicas, bicarbonatadas cálcicas e mistas (figura 3 e 4 ).

Destaca-se que as três classes de águas refletem a distribuição regional dos pontos. Os pontos no alto Salitre tiveram as águas classificadas como Cloretadas cálcicas, as do médio como mistas e as do baixo salitre como bicarbonatadas cálcicas.

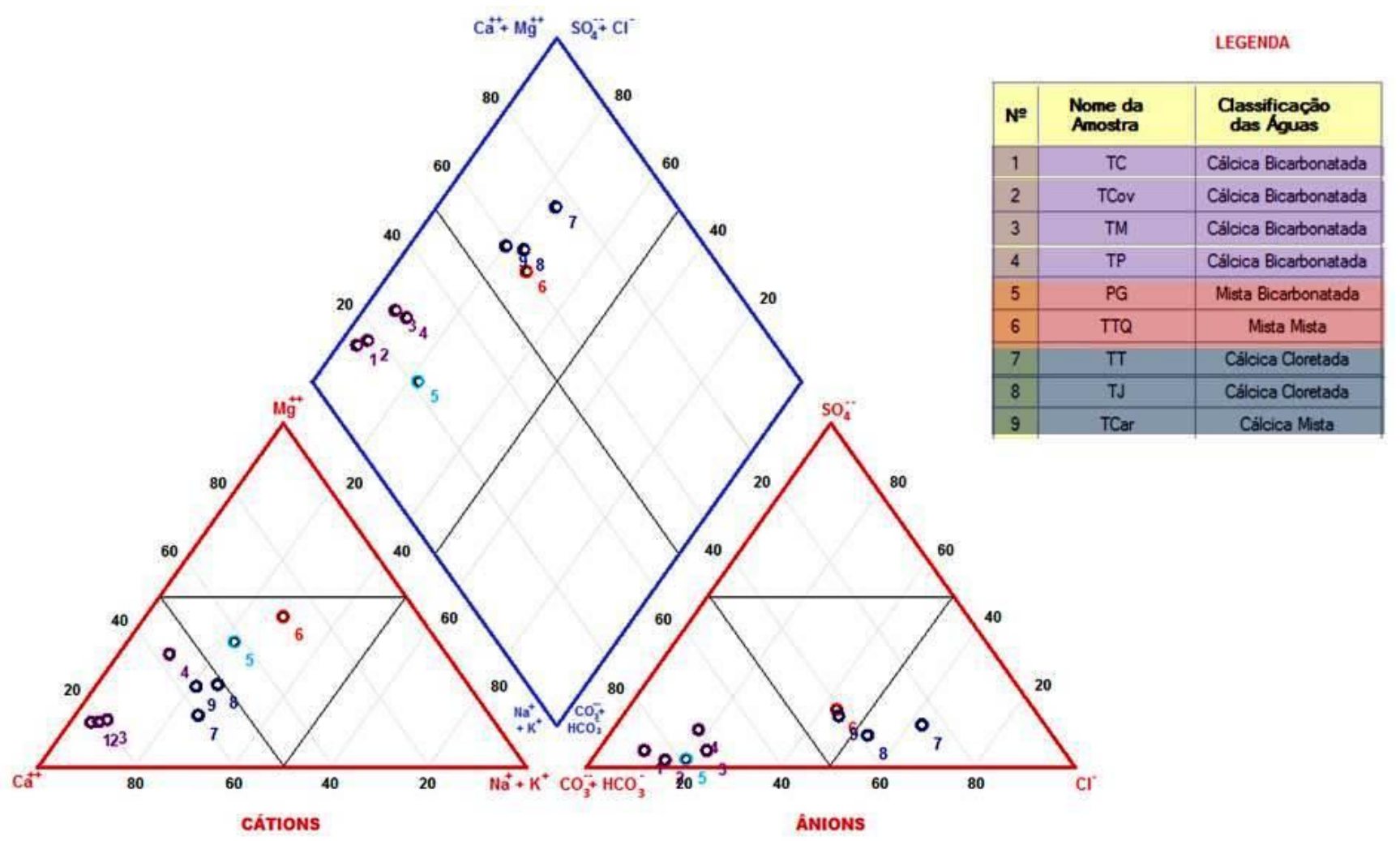

Figura 3. Diagrama de Piper para as águas subterrâneas encontradas nas cavernas da bacia do rio Salitre.

O diagrama de Stiff permite visualizar como as águas dos pontos amostrados podem ser agrupadas em três grupos de acordo com a posição do ponto na bacia. TC, TCov e TM são águas bicarbonatadas cálcicas do baixo Salitre; TP, TTiq e PG são as águas mistas encontrado na subregião do médio Salitre e TT, TJ e TCar são águas cloretadas cálcicas do alto Salitre. 

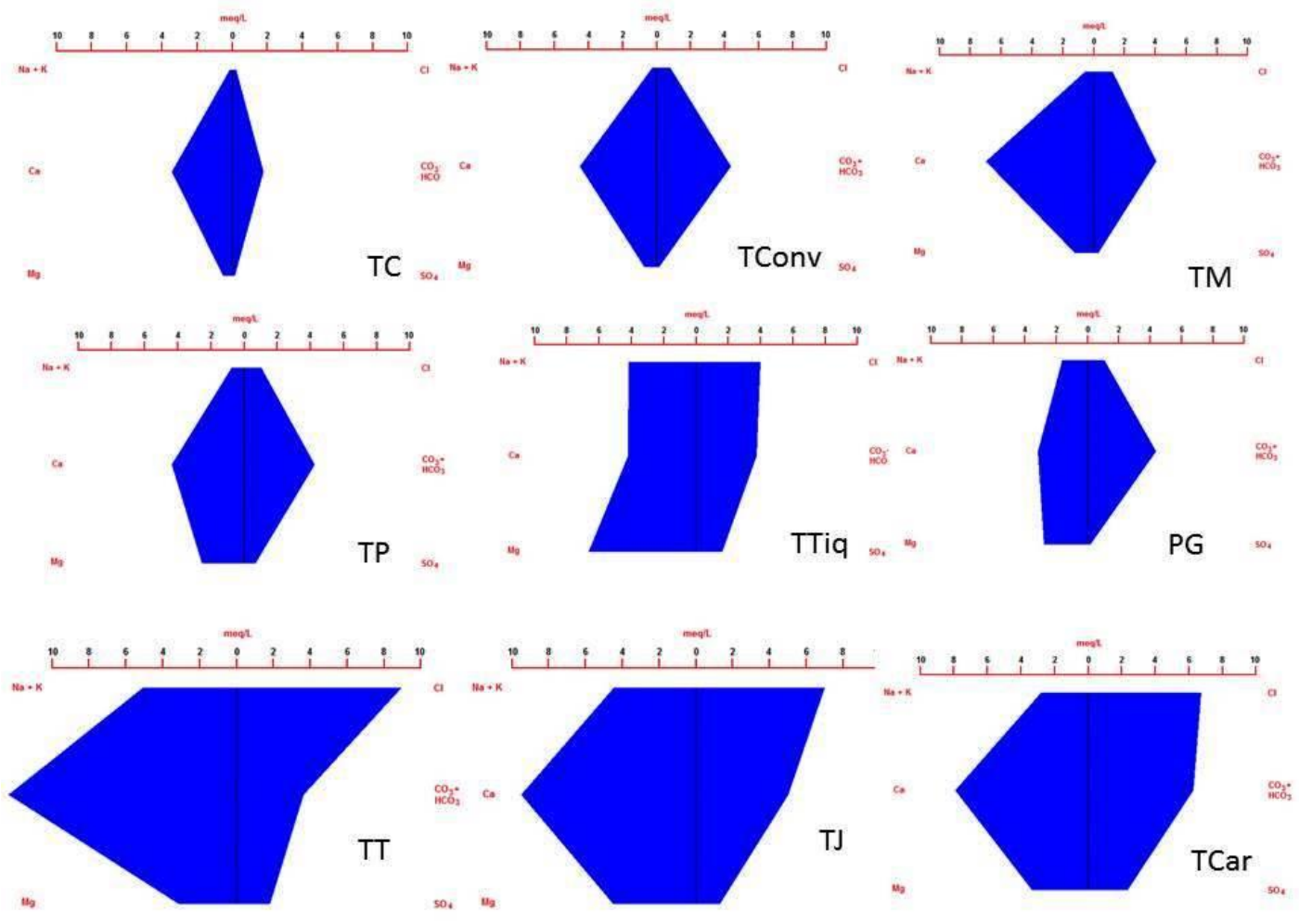

Figura 4. Diagrama de Stiff para as águas subterrâneas encontradas nas cavernas da bacia do rio Salitre.

\section{DADOS HIDROGEOLÓGICOS}

O aquífero cárstico do Grupo Una ocupa quase toda porção central do vale do rio Salitre. É o principal aquífero do vale do rio Salitre, e onde se encontram as principais reservas de águas subterrâneas.

Os processos de carstificação atuaram nas rochas carbonáticas do Una diferencialmente, tanto lateralmente como em profundidade, tendo como resultado um aquífero muito heterogêneo e anisotrópico, onde as suas características hidrogeológicas variam muito, tanto localmente como em nível regional, o que torna muito difícil caracteriza-lo do ponto de vista hidrodinâmico (Ribeiro, 2015).

Dois mapas de fluxo foram elaborados pelo método de krigagem levando em consideração a topografia e a condutividade elétrica (Figura 5). Nestes mapas foram plotadas a direção e sentido provável de circulação das águas subterrâneas. 

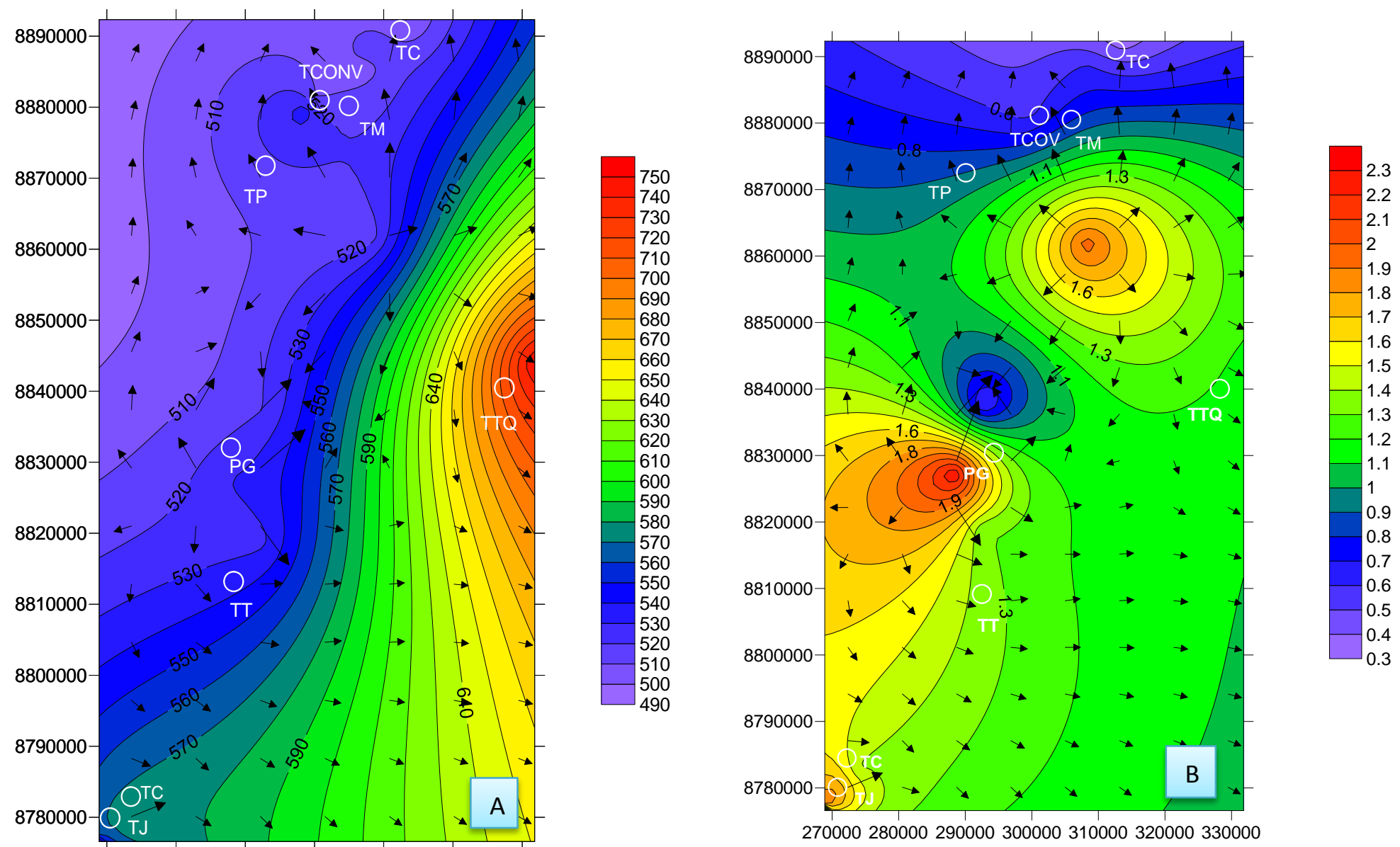

270000280000290000300000310000320000330000

Figura 5. Mapa de fluxo do aquífero cárstico na bacia do rio Salitre. (A) cota altimétrica. (B) Condutividade elétrica.

\section{DADOS BIOLÓGICOS}

Segundo (Koenemann \& Holsinger 2000) S. trajanoae foi registrado nas seguintes cavernas da bacia do rio Salitre: Toca do Convento, Toca do Pitu, Toca do Gonçalo e Toca do Teodoro. Araújo \& Peixoto (2015) registraram a ocorrência de S. trajanoae na Toca da Tiquara. O presente trabalho registra mais dois novos hábitats para $S$. trajanoae. Toca do Carlito e Toca da Jurema. Esse é o registro mais ao norte do gênero na bacia do Salitre.

As espécies do genero Spelaeogammarus estão distribuidas em uma série de cavernas em áreas cársticas descontinua ao longo de uma distancia linear de cerca de $1200 \mathrm{~km}$, de norte ao sul da Bahia (Figura 6). Os registros para cada espécie por área cárstica são: $S$. santanensis -1 caverna, $S$. bahiensis 1 caverna, S. spinilacertus - 2 cavernas, S. titan- 1 caverna, S. sanctum 1 caverna e $S$. trajanoae - 7 cavernas. 


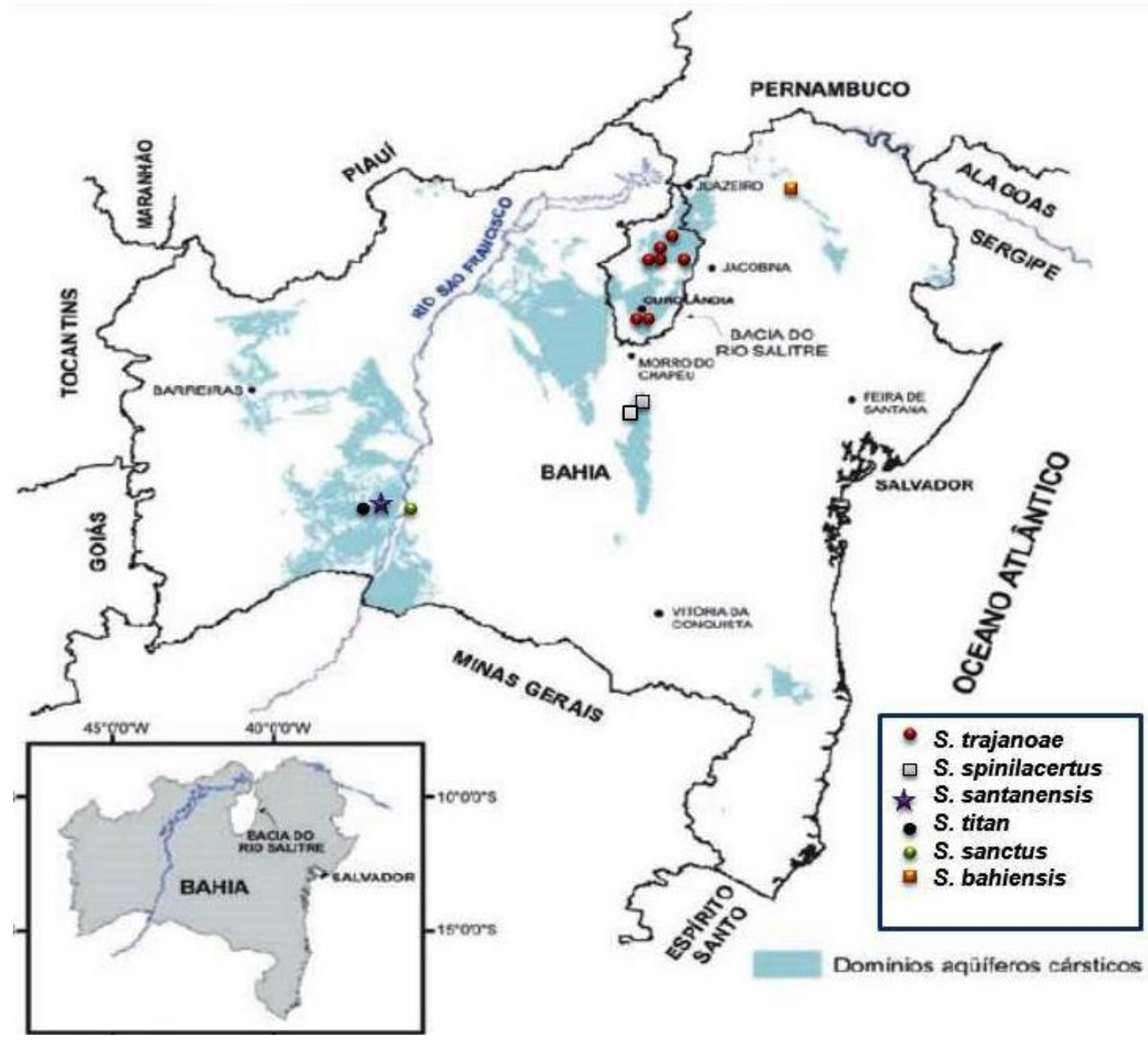

Figura 6. Distribuição geográfica do gênero Spelaeogammarus (Adaptado de Bastos-Pereira e Ferreira 2015).

\section{CONSIDERAÇÕES FINAIS}

Apesar do domínio aquífero cárstico na bacia do Salitre apresentar três regiões distintas com relação aos aspectos hidroquímicos, este fato parece não influenciar na distribuição da espécie S. trajanoae uma vez, que foi possível registrar exemplares dessa espécie em cavernas do alto, médio e baixo salitre.

Sendo uma espécie estritamente subterrânea a presença de $S$. trajanoae em cavernas ao longo do aquífero pode indicar uma conectividade. Os mapas de fluxo mostram uma circulação das 
águas subterrâneas congruente com a distribuição das espécies. Com uma direção geral do fluxo feita para a calha principal do rio Salitre.

Apesar de a espécie ser registrada em uma faixa ampla da bacia, cabe ressaltar que ela não foi encontrada em todas as cavernas amostradas. A ausência de S. trajanoae em cavernas próximas as que já foram registrados o espécime pode indicar que aquela caverna pode receber outra fonte de água. É possível também que outros fatores causaram a ausência (a espécie não estava presente no momento da amostragem ou há alguma limitação física ou química que impede a espécie de migrar até aquela caverna).

Futuros trabalhos com dados moleculares entre as espécies $S$. trajanoae, S. spinilacertus e S. bahiensis podem ajudar na elaboração de um modelo hidrogeológico para essas áreas cársticas atualmente disjuntas.

\section{REFERÊNCIAS BIBLIOGRÁFICAS}

ARAÚJO, A.V. \& PEIXOTO, R.S. 2015. The Impact of Geomorphology and Human Disturbances on the Faunal Distribution in Tiquara and Angico Caves of Campo Formoso, Bahia, Brazil. Ambient Science, 2014: http://www.caves.res.in/journal/articles/Amb_Sci_02(1)_Ra_04.pdf

ARIETTI, M. \& EDWARDS, B. 2006. Groundwater invertebrates in chalkboreholes and their significance in public water supply. Internal report for Three Valleys Water. $40 \mathrm{pp}$.

BASTOS-PEREIRA， R. \& FERREIRA R.L., 2015. A new species of Spelaeogammarus (Amphipoda: Bogidielloidea: Artesiidae) with an identification key for the genus. Zootaxa 4021 (3): 418-432. http://dx.doi.org/10.11646/zootaxa.4021.3.2.

BONACCI O, PIPAN T, CULVER D. 2009. A framework for karst ecohydrology. Environmental Geology 56: 891-900. DOI: 10.1007/ s00254-008-1189-0

DOLE- OLIVER, M. J. \& MARMONIER, P. 1992. Ecological requirements of stygofauna in an active channel of the Rhone River. Stygologia 7 (2), 65-75. 
GOLDSCHEIDER N, MEIMAN J, PRONK M, SMART C. 2008. Tracer test in karst hydrogeology and speleology. International Journal of Speleology 37: 27-40. $\underline{\text { http://scholarcommons.usf.edu/cgi/viewcontent.cgi?article }=1175 \& \text { context }=i j s}$

HAHN, H. J. 2006. The GW-Fauna-Index: a first approach to a quantitative ecological assessment of groundwater habitats. Limnologica $36 \quad$ (2), 119-137. http://www.sciencedirect.com/science/article/pii/S0075951106000181

KOENEMANN, S. \& HOLSINGER, J.R. 2000. Revision of the subterranean amphipod genus Spelaeogammarus (Bogidiellidae) from Brazil, including descriptions of three new species and considerations of their phylogeny and biogeography. Proceedings of the Biological Society of Washington, 113 (1), 104-123. http://biostor.org/reference/81035

MAURICE, L., BAKER, J.A., ATIKINSON, T.C., WILliAMS, A.T. \& SMART, P.L. 2011. A tracer methodology for identifying flow in and across boreholes. Ground Water 49 (2), 227-238. http://www.ncbi.nlm.nih.gov/pubmed/20477879

PIPAN, T. \& CULVER, D.C. 2007. Copepod distribution as an indicator of epikarst system $\begin{array}{lllll}\text { connectivity. } & \text { Hydrogeology } & \text { Journal } & \mathbf{1 5}(\mathbf{4}), & \text { 817-822. }\end{array}$ http://link.springer.com/article/10.1007\%2Fs10040-006-0114-4

RIBEIRO, S. H. S. Estudo das águas subterrâneas da bacia do rio Salitre, Bahia. Salvador. 2005. 109f. Monografia (Graduação)-Instituto de Geociências, Universidade Federal da Bahia, Salvador, 2005.

SILVA, A.B. 2002. Hidrogeologia de Meios Cársticos. Belo Horizonte: Universidade Federal de Minas Gerais, 39p, il.

SILVA, E. J. Caracterização Hidrogeológica de bacias hidrográficas do Semiárido baiano através de Técnicas de Geoprocessamento. 2009. 142 f. Dissertação de mestrado. Universidade Federal do Ceará - UFC, 2009.

SMART C, WORTHINGTON SRH. 2004. Water tracing. In Encyclopedia of Caves and Karst Science, Gunn J (ed). Fitzroy Dearborn: New York; 777-779. 